\title{
Nonlinear dynamics of confined thin liquid-vapor bilayer systems with phase change
}

\author{
Kentaro Kanatani ${ }^{1, a)}$ and Alexander Oron ${ }^{2, b)}$ \\ ${ }^{1}$ Graduate School of Engineering, \\ Yokohama National University, 79-5 Tokiwadai, Hodogaya, Yokohama 240-8501, Japan \\ ${ }^{2}$ Department of Mechanical Engineering, Technion-Israel Institute of Technology, Haifa 32000, Israel
}

(Received 7 November 2010; accepted 8 February 2011; published online 8 March 2011)

\begin{abstract}
We numerically investigate the nonlinear evolution of the interface of a thin liquid-vapor bilayer system confined by rigid horizontal walls from both below and above. The lateral variation of the vapor pressure arising from phase change is taken into account in the present analysis. When the liquid (vapor) is heated (cooled) and gravity acts toward the liquid, the deflection of the interface monotonically grows, leading to a rupture of the vapor layer, whereas nonruptured stationary states are found when the liquid (vapor) is cooled (heated) and gravity acts toward the vapor. In the latter case, vapor-flow-driven convective cells are found in the liquid phase in the stationary state. The average vapor pressure and interface temperature deviate from their equilibrium values once the interface departs from the flat equilibrium state. Thermocapillarity does not have a significant effect near the thermodynamic equilibrium, but becomes important if the system significantly deviates from it. () 2011 American Institute of Physics. [doi:10.1063/1.3559945]
\end{abstract}

\section{INTRODUCTION}

Phase change in the form of evaporation or condensation is a ubiquitous phenomenon in nature and various technological processes and devices, such as surface coating, drying of paint, and heat pumps, thin-film evaporators, condensers, and others. Therefore, modeling and investigation of phasechange processes is of great importance.

The effect of phase change often affects the stability of a liquid-gas interface. ${ }^{1,2}$ Linear stability analysis of the physical system consisting of a single fluid layer exposed to the passive air environment and restricted to monotonic stability only was carried out by Palmer. ${ }^{3} \mathrm{He}$ found that the liquid phase is unstable due to disturbances of the mass flux, interface deformation, vapor recoil for fast evaporation, and liquid flows sustained by the interfacial shear stress imparted by the vapor.

Prosperetti and Plesset ${ }^{4}$ revisited the linear stability analysis of an evaporating layer but this time for an inviscid fluid. They concluded that among the destabilizing effects proposed by Palmer, ${ }^{3}$ all but two, namely, those of viscosity and surface-tension gradient, are negligible. A possibility of the emergence of oscillatory instability was also suggested. They also showed that instability for a rapidly evaporating liquid sets in for a wide range of disturbance wavenumbers. Linear stability analysis of a viscous evaporating liquid layer based on the assumption of a small ratio between the vapor and liquid densities and using this ratio as a small perturbation parameter was carried out by Higuera. ${ }^{5}$

Nonlinear stability analysis of a thin liquid film exposed to the passive air ambience subjected to evaporation or condensation was first proposed by Burelbach et al. ${ }^{6}$ Their

\footnotetext{
${ }^{a)}$ Electronic mail: kentaro@ynu.ac.jp.

${ }^{b)}$ Electronic mail: meroron@techunix.technion.ac.il.
}

theory is based on the assumption that the density, dynamic viscosity, and thermal conductivity of the gas are much smaller than those of the liquid. This enabled them to effectively decouple the dynamics of the two phases and to consider that of the liquid only. This is the reason for the classification of this model as a "one-sided" approach. The only coupling between the two phases is the mass flux obtained from the Hertz-Knudsen law. ${ }^{3,7}$ Burelbach et al. ${ }^{6}$ modified the latter to the linearized form of the constitutive equation relating the mass flux and the temperature jump between the local interfacial temperature and the saturation temperature in the gas phase for the given pressure. The main feature found was that evaporation enhances instability triggered by other effects, such as thermocapillarity, while condensation contributes to stabilization of the film interface. ${ }^{6}$ The effect of vapor recoil was also incorporated into the model equation. It was found that vapor recoil is destabilizing in both evaporation and condensation. Based on the one-sided theory, Oron $^{8}$ showed that in the case of the layered attractive-repulsive potential for the intermolecular forces and moderate evaporation, a three-dimensional film undergoes the process of coarsening before flattening and subsequent disappearance. Two-dimensional evolution of evaporating and condensing liquid films was studied by Oron and Bankoff ${ }^{9,10}$ in the framework of the one-sided theory.

Sultan et al. ${ }^{11}$ developed a "two-sided" extension of the theory of evaporation by accounting for diffusion of vapor in the static gas phase. In this "diffusion-limited" regime, their linear stability analysis ${ }^{11}$ predicts the stabilization of longwavelength disturbances of the interface by evaporation.

Bestehorn and Merkt ${ }^{12}$ considered phase-change process in a thin liquid film suspended on a cooled horizontal substrate in the gravity field. They related the mass flux through the interface determined by Hertz-Knudsen law to the deviation of the local film thickness from the flat base state. Using 
this theory, Bestehorn ${ }^{13}$ numerically investigated the system with stable stratification heated from above in the threedimensional case and found that instead of drop coarsening in the nonvolatile case the presence of phase change may lead to formation of hexagonal and square patterns.

In the above-mentioned studies, the vapor phase was assumed to be infinitely deep. Huang and Joseph ${ }^{14}$ considered the stability of a bounded bilayer system with phase change, where several interfacial conditions were examined. Later, more general interfacial conditions allowing for nonequilibrium effects were applied to an evaporating bilayer system heated at the liquid side by Margerit et al. ${ }^{15}$ They demonstrated that the perturbation equations for a bilayer system can be reduced to those for a one-layer system using an equivalent Biot number. The evaporation was shown to be stabilizing for the interface, while the interfacial nonequilibrium has a destabilizing impact. They also found that discontinuity of the temperature at the interface measured recently ${ }^{16,17}$ affects only marginally the rate of the evaporation and the stability properties of the interface.

Furthermore, Ozen and Narayanan ${ }^{18,19}$ developed linear and weakly nonlinear stability theories for this physical setting. They found that in the case of evaporation, vapor flow stabilizes the interface, whereas flow in the liquid phase promotes its destabilization, if the system is heated on the liquid side. ${ }^{18}$ Their weakly nonlinear theory ${ }^{19}$ revealed that instability sets in via supercritical bifurcation and stressed the importance of accounting for the vapor dynamics. Ozen and Narayanan ${ }^{20}$ also studied the influence of evaporation on the stability of the unstable stratified vapor-liquid system and concluded that phase change imparts a stabilizing impact on the Rayleigh-Taylor instability. Their work was followed by McFadden et $a l^{21}$ and Guo and Narayanan, ${ }^{22}$ where the same trend of the results was found. McFadden and Coriell ${ }^{23}$ reconsidered the bilayer problem studied by Huang and Joseph $^{14}$ with cooling at the liquid side and found oscillatory instability as reported in Ref. 14.

Recently, Kanatani ${ }^{24}$ developed a two-layer theory for a confined thin bilayer system by applying long-wave approximation $^{1}$ to both liquid and vapor layers. There have been numerous studies on bounded bilayer films. ${ }^{2,25-27}$ However, the distinct difference of the system in Ref. 24 is the presence of large disparity in material properties between liquid and vapor. Assuming that the ratio of the vapor density to that of the liquid is much smaller than the corresponding ratio for the dynamic viscosities, which in turn is much smaller than unity, he showed that the vapor pressure effect dominates over other effects such as mass loss/gain or vapor recoil. In this system, temperature fluctuations at the liquidvapor interface induce lateral variation of the vapor pressure due to the effect of phase change: the vapor pressure becomes higher where the liquid evaporates and lower where the vapor condenses. Consequently, the higher vapor pressure pushes the interface and the lower one pulls it. Therefore, the effect of the vapor pressure is destabilizing when the liquid side is heated and stabilizing when the vapor side is heated. Here, the vapor pressure serves as an active source of the liquid dynamics. This vapor-pressure-induced mechanism was not considered in bilayer systems without phase change, ${ }^{28-31}$ where the gas dynamics was ignored and only the heat conduction in the gas phase was considered. Strictly speaking, the variation of the vapor pressure was taken into account in the model of film boiling. ${ }^{32}$ However, its effect on the dynamics of the liquid-vapor interface was not sufficiently accounted for there.

In this paper, we numerically investigate the nonlinear evolution of this system, following our previous linear stability analysis. ${ }^{24}$ In Ref. 24 , the two cases were addressed: the first is that of the liquid (vapor) heated (cooled) and gravity acting toward the liquid (superheated or supercooled case), and the second is of the liquid (vapor) cooled (heated) and gravity acting toward the vapor (Rayleigh-Taylor unstable case). Both of these cases are also considered in this work. We discuss the possibility of the emergence of nontrivial nonruptured stationary states. The difference from the other Marangoni-driven bilayer systems ${ }^{28-31}$ and "thick" bilayer systems with phase change $e^{18,19,22,33}$ is emphasized.

The structure of the paper is as follows. Section II presents the mathematical framework of the investigation. Section III describes the numerical methods used for solving the governing equations. Section IV presents the results of the computations for the two cases mentioned above. Section V contains a discussion on the obtained behavior of the vapor pressure. Section VI reveals the effect of thermocapillarity and its connection with the degree of the interfacial nonequilibrium. Section VII contains the summary and conclusions. The Appendix complements the linear stability analysis made in Ref. 24 for the case where the system significantly deviates from thermodynamic equilibrium and the Marangoni effect is sizeable.

\section{GOVERNING EQUATIONS}

A two-dimensional bilayer liquid-vapor system bounded by two horizontal planar solid surfaces in the gravity field is considered in this paper. A thin liquid layer is assumed to be initially in thermodynamic equilibrium with its vapor layer, so that the liquid-vapor interface is at the saturation temperature $T_{\text {sat. }}$. The temperatures of the liquid-side and vapor-side surfaces are maintained at constant $T_{1}$ and $T_{2}$. The $x$ axis is chosen along the liquid-solid boundary, whereas the $z$ axis is perpendicular to it and points into the system. The liquidvapor interface is located at $z=h(x, t)$ and varies in both space and time.

Application of the long-wave theory to both liquid and vapor layers ${ }^{24}$ yields the following two dimensionless equations for each layer:

$$
\begin{aligned}
& \partial_{t} h=-\partial_{x} q_{l}, \\
& E J=\rho \partial_{x} q_{v},
\end{aligned}
$$

where $J, q_{l}$, and $q_{v}$ are the mass flux through the interface due to evaporation and condensation, and the volumetric flow rates of the liquid and vapor layers, respectively, expressed by 


$$
\begin{aligned}
& J=-\frac{K}{1+K h+\frac{\lambda h}{1+d-h}} \\
& \times\left[\frac{(1+d)(h-1)}{1+d-h}+\left(1+\frac{\lambda h}{1+d-h}\right) \frac{\Pi}{\rho E} p_{v}\right], \\
& q_{l} \equiv \int_{0}^{h} u_{l} d z \\
&=-\frac{h+3(1+d)}{12} h^{2} \partial_{x} p_{v}-\frac{h^{3}}{3} \partial_{x}\left(G h-S \partial_{x}^{2} h\right)-\frac{h^{2}}{2} M \partial_{x} T_{I},
\end{aligned}
$$

$$
q_{v} \equiv \int_{h}^{1+d} u_{v} d z=-\frac{1}{12 \eta}(1+d-h)^{3} \partial_{x} p_{v}
$$

Here, $u_{l}$ and $u_{v}$ are the horizontal components of the liquid and vapor velocities, $p_{v}$ is the vapor pressure, and $T_{I}$ is the interface temperature given by

$$
T_{I}=\frac{1}{1+K h+\frac{\lambda h}{1+d-h}}\left[-\frac{(1+d)(h-1)}{1+d-h}+\frac{K \Pi}{\rho E} h p_{v}\right] \text {. }
$$

The dimensionless scaled parameters $G, S, M, E, \Pi, K, \rho, \eta$, $\lambda$, and $d$ represent gravity, surface tension, thermocapillarity, rate of evaporation (condensation), rate of saturation temperature variation with respect to the vapor pressure, deviation from thermodynamic equilibrium, density ratio, dynamic viscosity ratio, thermal conductivity ratio, and initial thickness ratio, respectively, and are defined by

$$
\begin{aligned}
& G=\frac{\epsilon g d_{l}^{3} \rho_{l}^{2}}{\eta_{l}^{2}}, \quad S=\frac{\epsilon^{3} \sigma_{0} \rho_{l} d_{l}}{\eta_{l}^{2}}, \quad M=\frac{\epsilon d_{l} \rho_{l} \gamma \Delta T}{\eta_{l}^{2}}, \\
& E=\frac{\epsilon^{-2} \lambda_{l} \Delta T}{\eta_{l} L}, \quad \Pi=\frac{\epsilon^{-5} \lambda_{l} \eta_{l} T_{\mathrm{sat}}}{\left(L d_{l} \rho_{l}\right)^{2}}, \\
& K=\alpha \frac{\rho_{v} d_{l} L^{2}}{\lambda_{l} T_{\mathrm{sat}}} \sqrt{\frac{m}{2 \pi k_{B} T_{\mathrm{sat}}}}, \quad \rho=\frac{\epsilon^{-2} \rho_{v}}{\rho_{l}}, \quad \eta=\frac{\epsilon^{-1} \eta_{v}}{\eta_{l}}, \\
& \lambda=\frac{\lambda_{v}}{\lambda_{l}}, \quad d=\frac{d_{v}}{d_{l}},
\end{aligned}
$$

where $\epsilon$ is a small parameter defined as the ratio between the mean thickness of the liquid layer and the typical wavelength of the longitudinal disturbance. In the above definitions, $\rho_{\beta}, \eta_{\beta}, \lambda_{\beta}$, and $d_{\beta}$ denote the density, dynamic viscosity, thermal conductivity, and mean thickness, respectively, in the $\beta=\{v, l\}$ phase, where $v$ denotes the vapor and $l$ the liquid; $g$ is the gravitational acceleration; $L$ is the latent heat of vaporization; $\alpha$ is the accommodation coefficient; $m$ is the molecular mass of the fluid; and $k_{B}$ is the Boltzmann constant. The surface tension $\sigma$ has a linear dependence on the temperature,

$$
\sigma=\sigma_{0}-\gamma\left(T_{I}-T_{\mathrm{sat}}\right)
$$

where $T_{\text {sat }}$ is related to the wall temperatures $T_{1}$ and $T_{2}$ by

$$
T_{\mathrm{sat}}=\frac{\lambda T_{2}+d T_{1}}{\lambda+d}
$$

and $\sigma_{0}$ is the reference value of surface tension at this temperature. $\Delta T$ is the initial temperature difference across the liquid layer,

$$
\Delta T \equiv T_{1}-T_{\mathrm{sat}}=\frac{\lambda}{\lambda+d}\left(T_{1}-T_{2}\right)
$$

which is positive (negative) for the case of the heated (cooled) liquid-solid boundary.

The dimensionless variables $h, x, p_{v}, T_{I}, t$, and $J$ are in units of $d_{l}, d_{l} / \epsilon, \eta_{l}^{2} / \epsilon d_{l}^{2} \rho_{l}, \Delta T, d_{l}^{2} \rho_{l} / \epsilon \eta_{l}$, and $\lambda_{l} \Delta T / d_{l} L$, respectively. The values of the dimensionless vapor pressure and interface temperature are set to 0 at the initial equilibrium state.

Equations (1a) and (1b) are obtained from integration of the continuity equations in the liquid and vapor phases in the $z$ direction over each layer under the appropriate boundary conditions. Note that Eq. (1a) is of the standard massconservative form, although both evaporation and condensation take place between the liquid and vapor phases. This is because we have neglected the term of mass loss or gain (cf. Ref. 6, Ref. 12, or Ref. 13) compared to the other terms in Eq. (1a) (for details, see Ref. 24). Equation (1b) shows that the mass flux due to phase change (evaporation and condensation) induces the vapor flow. As it follows from Eq. (2a), the mass flux arises from the effect of the interface deformation (the first term in the square brackets) and that of variation of the saturation temperature due to the vapor pressure (the second term). Note that the mass flux is related to the interface temperature by $J=K\left(T_{I}-\Pi p_{v} / \rho E\right)$ [Eq. (27h) of Ref. 24].

Substituting Eq. (2) into Eq. (1) results in the following set of equations:

$$
\begin{aligned}
\partial_{t} h= & \partial_{x}\left[\frac{h+3(1+d)}{12} h^{2} \partial_{x} p_{v}+\frac{h^{3}}{3} \partial_{x}\left(G h-S \partial_{x}^{2} h\right)\right. \\
& \left.+\frac{h^{2}}{2} M \partial_{x} T_{I}\right], \\
\frac{E(1+d)(h-1)}{1+d-h}+\frac{\Pi}{\rho}\left(1+\frac{\lambda h}{1+d-h}\right) p_{v} & \\
= & \frac{\rho}{12 \eta} \frac{1+K h+\frac{\lambda h}{1+d-h}}{K} \partial_{x}\left[(1+d-h)^{3} \partial_{x} p_{v}\right],
\end{aligned}
$$

which describes the spatiotemporal evolution of the two unknown variables $h(x, t)$ and $p_{v}(x, t)$ with $T_{I}(x, t)$ expressed via them by Eq. (3). The first term in the right-hand side of Eq. (8a) accounts for the effect of lateral vapor pressure variation, the second term there describes the combined effects of gravity and surface tension, while the last one of Eq. (8a) represents the Marangoni effect. 
Linear stability of the base state that consists of a flat interface $h \equiv 1$ and a uniform vapor pressure $p_{v} \equiv 0$ of Eq. (8) was investigated in detail in Ref. 24. The growth rate $\omega$ of an infinitesimal disturbance with the wavenumber $k$ is given by the dispersion relation

$$
\omega=\frac{A k^{2}}{k^{2}+k_{0}^{2}}-\frac{1}{3} k^{2}\left(G+S k^{2}\right)+\frac{1+d}{2 d} \frac{M k^{2}}{1+K+\frac{\lambda}{d}},
$$

with

$$
\begin{aligned}
& A=\frac{\eta(1+d)}{\rho d^{4}} \frac{K}{1+K+\frac{\lambda}{d}}\left[(4+3 d) E+\frac{6 M \Pi}{\rho} \frac{K}{1+K+\frac{\lambda}{d}}\right], \\
& k_{0}^{2}=\frac{12 \eta(d+\lambda)}{\rho^{2} d^{4}} \frac{K}{1+K+\frac{\lambda}{d}} \Pi,
\end{aligned}
$$

where positive (negative) values of $\omega$ indicate instability (stability) of the base state.

The main conclusions of the assessment of the linear stability for a water/vapor system at $373 \mathrm{~K}$ and 1 atm carried out in Ref. 24 are as follows. (i) Only slight temperature gradients are sufficient to overcome the stabilizing gravitational effect for the superheated or supercooled case when the liquid side is heated and gravity acts toward the liquid. (ii) The stabilizing vapor pressure effect and the destabilizing gravitational effect balance near $|\Delta T|=0.1 \mathrm{~K}, \quad d_{l}=1.0$ $\times 10^{-4} \mathrm{~m}$, and $d=2$ for the Rayleigh-Taylor unstable case where the liquid side is cooled and gravity acts toward the vapor. (iii) Thermocapillarity makes little contribution as compared to the other effects. However, as was already pointed out in Ref. 24, the last conclusion may not be the case if the accommodation coefficient is much less than 1. The contribution of the Marangoni effect for small accommodation coefficients is estimated in the Appendix. It is shown there that thermocapillarity becomes important in the limit of $\alpha \rightarrow 0$, and the critical condition reduces to that of the Rayleigh-Taylor unstable bilayer system without phase change. $^{29,30}$

Equations (8) and (3) are numerically investigated with periodic boundary conditions in terms of $h, T_{I}$, and $p_{v}$ in the domain $0 \leq x \leq l$ and the initial condition in the form

$$
h(x, t=0)=1+\delta \cos \left(\frac{2 n \pi x}{l}\right)
$$

where we set $l=2 \pi$ and $\delta=0.05$. The parameter $n$ in Eq. (11) indicates the wavenumber of the initial disturbance.

\section{NUMERICAL METHOD}

The set of Eqs. (8) and (3) is numerically solved using the method of finite differences where the NewtonKantorovich method is employed for time-marching in Eq. (8a). First, the vapor pressure and interface temperature are
TABLE I. Physical properties of water/vapor at $373 \mathrm{~K}$ and $1 \mathrm{~atm}$.

\begin{tabular}{lc}
\hline \hline$\rho_{l}=960 \mathrm{~kg} / \mathrm{m}^{3}$ & $\rho_{v}=0.6 \mathrm{~kg} / \mathrm{m}^{3}$ \\
$\eta_{l}=2.9 \times 10^{-4} \mathrm{~kg} / \mathrm{m} \mathrm{s}$ & $\eta_{v}=1.3 \times 10^{-5} \mathrm{~kg} / \mathrm{m} \mathrm{s}$ \\
$\lambda_{l}=6.8 \times 10^{-1} \mathrm{~J} / \mathrm{m} \mathrm{s} \mathrm{K}$ & $\lambda_{v}=2.5 \times 10^{-2} \mathrm{~J} / \mathrm{m} \mathrm{s} \mathrm{K}$ \\
$\kappa_{l}=1.7 \times 10^{-7} \mathrm{~m}^{2} / \mathrm{s}$ & $\kappa_{v}=2.0 \times 10^{-5} \mathrm{~m}^{2} / \mathrm{s}$ \\
$L=2.3 \times 10^{6} \mathrm{~J} / \mathrm{kg}$ & $\sigma_{0}=5.8 \times 10^{-2} \mathrm{~N} / \mathrm{m}$ \\
$\gamma=2.0 \times 10^{-4} \mathrm{~N} / \mathrm{m} \mathrm{K}$ & \\
\hline \hline
\end{tabular}

calculated from Eqs. (8b) and (3) for a given interface location at the previous time or initial condition. Based on these, the interface location at the new time is determined from Eq. (8a). More details on the numerical method are given in Refs. 34 and 35. Equation (8a) is rewritten in the form $h_{t}+F(h)=0$ and linearized using the Frechet derivative of the operator $F$. Evaluation of the Frechet derivatives and the finite difference discretization is carried out in the conservative form using linear interpolation for half-nodes, so that the total film mass is conserved up to $\mathcal{O}\left(10^{-12}\right) \%$ of its initial value. The time-marching technique is a second-order Runge-Kutta method accurate to $\mathcal{O}\left(\Delta t^{2}\right)$ where the size of the time step is normally in the range of $10^{-4}-10^{-3}$. The equidistant grids of 800-1200 are normally used below to determine the solutions for the set of Eqs. (8) and (3) in a periodic domain. The sets of simultaneous linear algebraic equations that possess a pentadiagonal matrix with triangular corners, and a tridiagonal matrix with corners arising from discretization of Eqs. (8a) and (8b), respectively, with the periodic boundary conditions, are solved using the modified Thomas algorithm.

Note that this procedure effectively ignores the temporal variation of the vapor pressure and the interface temperature within each time step. However, this effect can be neglected if the variation is sufficiently slow. We confirmed this by decreasing the value of $\Delta t$ from that used in the computations presented below and verified that this does not affect the evolution of the system.

\section{RESULTS}

In our numerical investigation, we consider a water/ vapor bilayer system at the temperature of $373 \mathrm{~K}$ and a pressure of 1 atm. The physical properties of the liquid and vapor are given in Table I. We fix the liquid depth to $10^{-4} \mathrm{~m}$, so that the units of the dimensionless time, liquid thickness, and vapor pressure correspond to $3.3 \mathrm{~s}, 10^{-4} \mathrm{~m}$, and $8.8 \times 10^{-3} \mathrm{~Pa}$, respectively, in the real setting. The value of $\epsilon$ is set to $10^{-2}$ throughout this paper.

We first study a bilayer system with a stable stratification, namely, where the vapor phase is above the liquid phase, heated at the solid-liquid boundary, or equivalently cooled at the solid-vapor boundary. We refer to these settings as the superheated or supercooled state, respectively. We then consider the opposite case where the vapor phase is located underneath the liquid phase, so that Rayleigh-Taylor instability is combined with heat and mass transfer. 

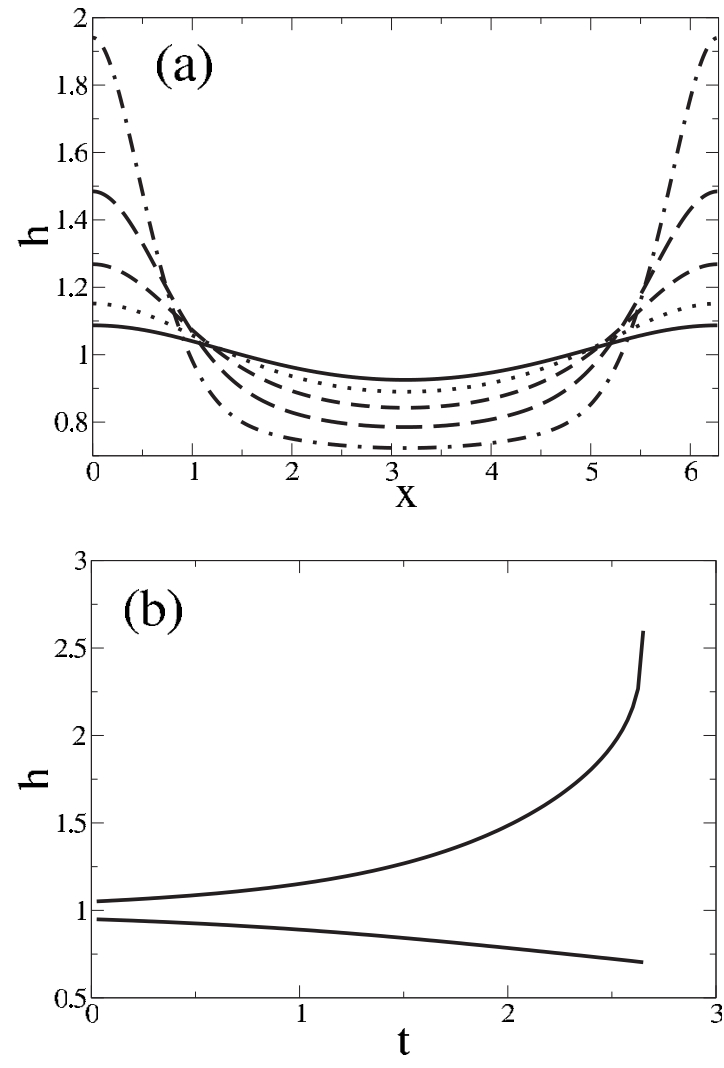

FIG. 1. Spatiotemporal evolution of the liquid film thickness. (a) Evolution of the liquid film thickness with time. The liquid phase is located under the corresponding curve. Curves from bottom to top at $x=0$ denote, respectively, the film thickness at $t=0.5,1,1.5,2$, and 2.5. (b) Time evolution of the maximal and minimal thicknesses of the liquid film. The curves terminate at the moment when the film interface touches the upper solid substrate.

\section{A. Superheated or supercooled state}

In this subsection, we present the results for the superheated or supercooled state for the parameter set of $E=1(\Delta T=0.1 \mathrm{~K}), g=9.8 \mathrm{~m} / \mathrm{s}^{2}, d=2, \alpha=1$, and $n=1$. Figure 1 illustrates the spatiotemporal evolution of the film thickness. The surface deflection grows monotonically until the crest of the liquid touches the ceiling; in other words, the vapor layer undergoes rupture. In contrast, the rupture of the liquid layer is not observed. We found that the rupture of the liquid layer does not take place in the range of $d \leq 5$. The absence of the liquid rupture in our system is supposedly due to the fact that in our case the vapor pressure pushes the round and wide trough of the interface in a distributed way. This mechanism is in sharp contrast with that of a Marangoni-driven bilayer system, ${ }^{28,30}$ where the surfacetension gradient pulls the liquid away from a narrow localized trough. In that case, the rupture of either the liquid or gas layer takes place depending on the system parameters.

Figure 2 demonstrates the velocity fields in both layers prior to the moment when the phase interface touches the upper substrate at $t=2.5$. The velocity field is determined using Eqs. (32)-(35) of Ref. 24 with the film thickness and the vapor pressure obtained from the numerical computation. In the figure, the vapor velocity field is compressed by the factor of $\epsilon$ as compared to the liquid velocity field [see the scalings (23) of Ref. 24]. Note that the $x$-component of the

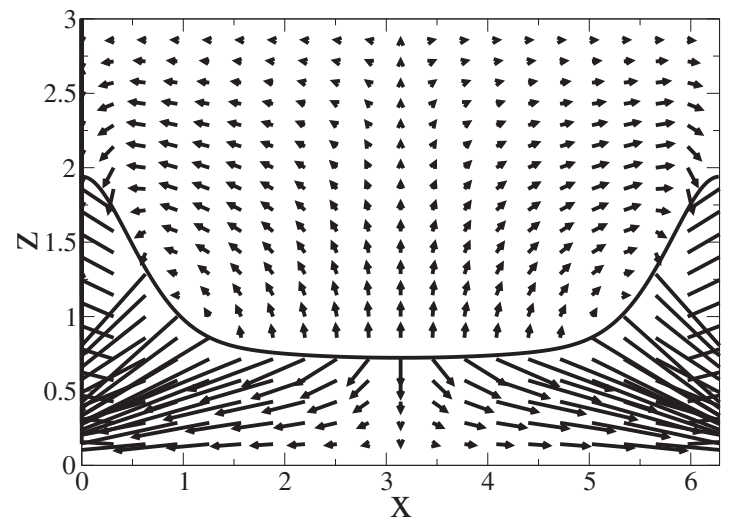

FIG. 2. Velocity field in both phases at $t=2.5$. The curve represents the liquid-vapor interface. The vapor velocity field shown here contains the factor of $\epsilon$ as compared to the liquid velocity.

velocity is also compressed by the factor of $\epsilon$, corresponding to the scaling of the $x$-coordinate mentioned in the paragraph following Eq. (7). From Fig. 2, it is obvious that the liquid is pushed downward at the trough and this velocity field is essentially different from that shown in Fig. 12 of Ref. 28, emphasizing the peculiarity of the instability mechanism for this system mentioned above. Based on the conservation of the liquid mass, its flow leads to the formation of the liquid crest. We also observe the vapor flow pattern emanating from the depressed evaporating portion of the interface and condensing onto the elevated part of the interface, thereby forming a pair of vortices.

Figure 3 displays the spatiotemporal evolution of the vapor pressure. As expected, the vapor pressure attains its maximum and minimum in the depressed and elevated por-
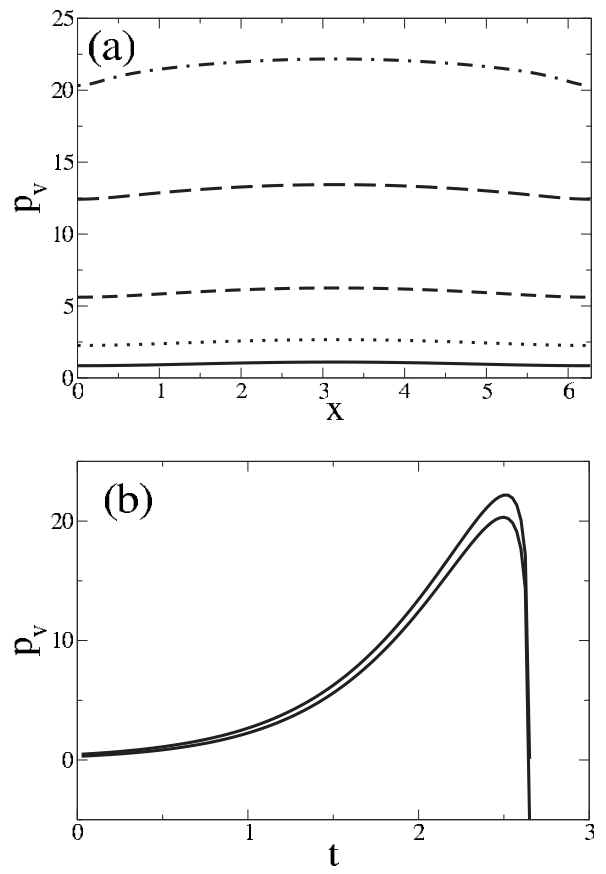

FIG. 3. Spatiotemporal evolution of the vapor pressure. (a) Variation of the vapor pressure with time where curves from bottom to top correspond to $t=0.5,1,1.5,2$, and 2.5 . (b) Temporal evolution of the maximal and minimal vapor pressures. 


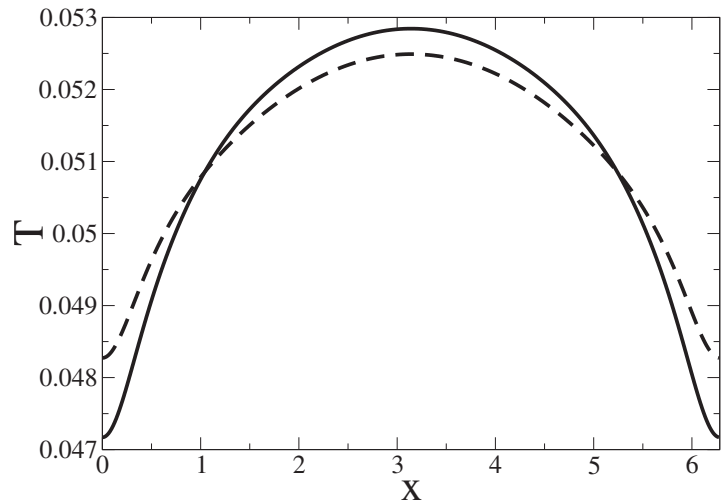

FIG. 4. Comparison between the interface temperature $T_{I}$ given by Eq. (3) (solid curve) and the saturation temperature variation $\Delta T_{s}\left(p_{v}\right)=(\Pi / \rho E) p_{v}$ (dashed curve) defined in Eq. (12) at $t=2.5$.

tions of the interface because of the effects of evaporation and condensation, respectively. We observe that the spatial average value of the vapor pressure deviates from zero, its equilibrium value, once the instability of the flat interface takes place. Initially, its value increases with time. However, its sudden drop occurs just before the phase interface touches the upper substrate, as seen in Fig. 3(b). We will discuss the reason for this behavior of the vapor pressure in Sec. V.

The interface temperature is determined from Eq. (3). Its spatiotemporal evolution is found to resemble that of the vapor pressure, and the reason for this can be deduced from the expression of the interface temperature, Eq. (3). Since $K=1.2 \times 10^{3}$ for the present system with $\alpha=1$, one can approximate Eq. (3) by

$$
T_{I} \simeq \frac{\Pi}{\rho E} p_{v} \equiv \Delta T_{s} .
$$

In the local thermodynamic equilibrium limit, $K \rightarrow \infty$, Eq. (12) holds exactly. The expression in the right-hand side of Eq. (12) represents the saturation temperature variation due to the vapor pressure fluctuation. Therefore, the physical meaning of Eq. (12) is that the interface temperature almost coincides with the saturation temperature variation. Figure 4 presents a comparison between them and shows that their difference is small. Hence, Eq. (12) provides a good approximation for the case shown in Fig. 4. In other words, the interface temperature is determined to a great extent by the variation of the saturation temperature or the vapor pressure, rather than by the interfacial deformation. We note that Eq. (12) becomes no longer valid for very small values of the accommodation coefficient, which will be shown in Sec. VI.

Figure 5 presents the spatiotemporal variation of the mass flux across the interface determined from Eq. (2a). Here, positive and negative values of $J$ correspond to evaporation and condensation, respectively. Comparing it with Fig. 1(a), we observe that evaporation and condensation occur in the trough and the crest, respectively, and their rates increase with the amplitude of the interface deflection. Note that the difference between the two curves in Fig. 4 is pro-

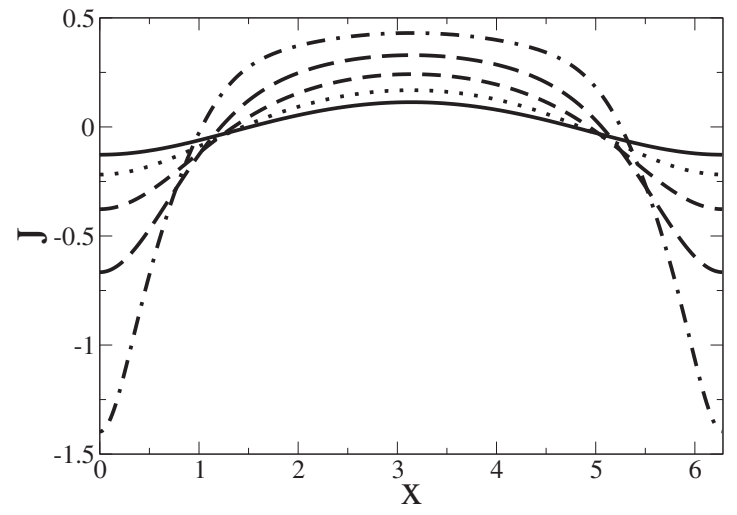

FIG. 5. Temporal evolution of the mass flux $J$ across the interface. Positive and negative values of $J$ correspond to evaporation and condensation, respectively. Curves from top to bottom at $x=0$ correspond to $t=0.5,1,1.5,2$, and 2.5 .

portional to the value of the mass flux. The total mass flux over the spatial period vanishes in conformance with Eq. (1b).

Summarizing this subsection, it is important to mention the possibility of the emergence of nonruptured steady states in the system discussed here. For a single thin liquid film with a standard thermocapillarity, it was shown analytically that nonruptured deflected stationary states of the system do not exist even in the presence of the stabilizing gravity effect. $^{36,37}$ Furthermore, the instability of a bilayer system without phase change is shown to be subcritical. ${ }^{28}$ It is of interest to look for steady states in our case in the linearly unstable domain. We numerically investigated the temporal evolution of the interface in our system near the linear stability threshold [Eq. (48) of Ref. 24] without the Marangoni effect for simplicity. We found that the maximal liquid thickness monotonically increases until the rupture of the vapor layer. Hence, nontrivial stationary states of the system based on Eq. (8) do not exist in the case where the temperature of the liquid-solid boundary is higher than that of the vaporsolid one. This result appears to be different from that of a weakly nonlinear analysis of a similar bilayer system which predicts a forward pitchfork bifurcation of the instability for a pure phase-change problem. ${ }^{19,22}$ However, the system discussed there is different from that considered here in that the liquid depth in their system is larger, so that small-scale convection takes place, which cannot be captured by the longwave theory applied here. Guo and Narayanan ${ }^{22}$ recently found subcritical bifurcation for a larger aspect ratio (the scaled width) of the container and a smaller scaled vapor depth when Rayleigh convection (buoyancy) is absent. They concluded that the Rayleigh convection in the liquid phase is responsible for the emergence of supercritical bifurcation, since in its presence subcritical bifurcation transforms to supercritical upon an increase in the liquid depth.

\section{B. Rayleigh-Taylor instability}

We next discuss the case where gravity acts toward the vapor, and therefore the system is subjected to RayleighTaylor instability. To accommodate this case into our model, 

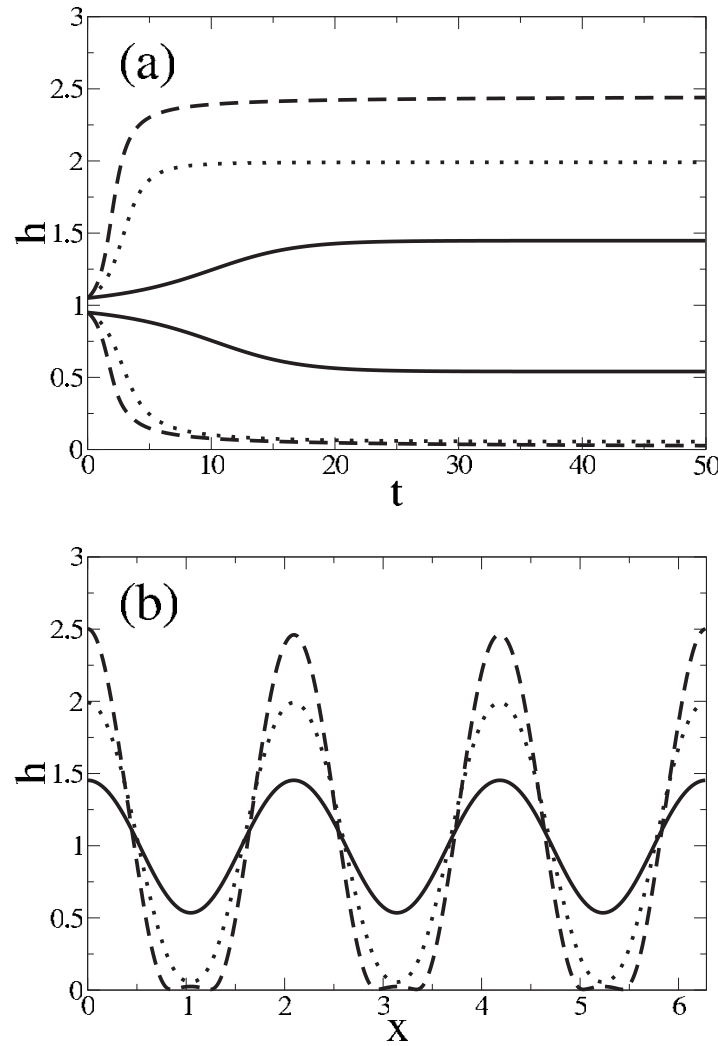

FIG. 6. Film evolution in the case of Rayleigh-Taylor instability for $d=2$ and $E=-1$ (solid curve), -0.5 (dotted curve), and -0.1 (dashed curve). (a) Temporal evolution of the maximal and minimal values of the film thickness. (b) The stationary states. The liquid phase is located under the corresponding curve, and gravity is directed upward.

we change the signs of both gravity and the temperature difference, so that the effects of vapor pressure and gravity counteract each other. As in Sec. IV A, we set $d=2$ and $\alpha=1$. For the initial condition, we consider a case of $n=3$ in Eq. (11) which corresponds to the fastest growing unstable mode for $n \in \mathbb{Z}$ for the Rayleigh-Taylor instability, see Fig. 4 of Ref. 24.

In the system subjected to the Rayleigh-Taylor instability, stationary states emerge following a long-time evolution, as shown in Fig. 6. Figures 6(a) and 6(b) present the temporal evolution of the maximal and minimal values of the film thickness, and the resulting stationary states, respectively, for various values of the evaporation number. In this subsection, negative values of $E$ imply heating from the vapor side. The amplitude of the stationary state of the system becomes larger as the absolute value of the evaporation number decreases. For $E=-0.1$, satellite drops emerge in the troughs of the liquid film. The minimal film thickness in this case is larger than 0.003 corresponding to $300 \mathrm{~nm}$, where the liquid layer is not ruptured by attractive van der Waals forces based on the criterion of Ref. 30. For other values of $E$ between -0.1 and -1 , a similar temporal evolution was found, and the maximal and minimal values of the film thickness depend monotonically on the value of $E$.

Figure 7 presents the velocity field of the steady state in one spatial period, i.e., one third of the computational domain. As in Fig. 2, we have scaled the vapor velocity by $\epsilon$. (a)

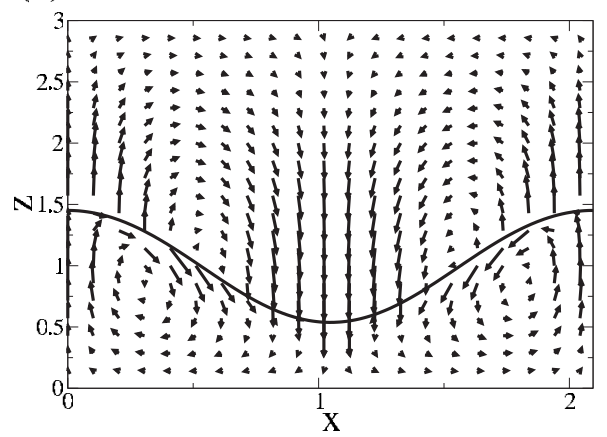

(b)

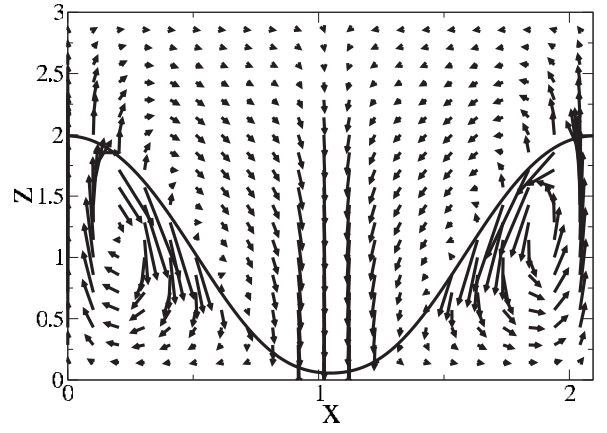

(c)

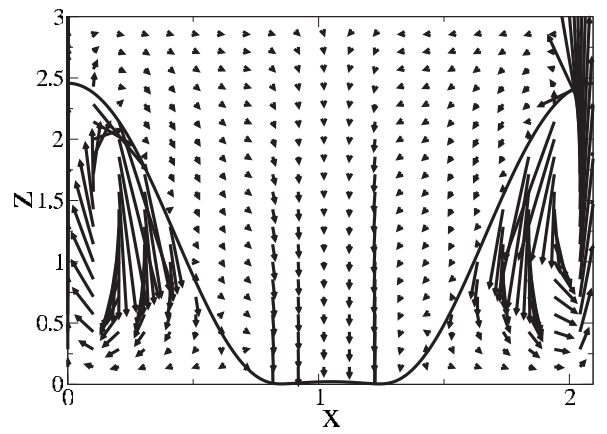

FIG. 7. Velocity field in the stationary state of the system. (a) $E=-1$, (b) $E=-0.5$, and (c) $E=-0.1$. The curve in each of the panels represents the liquid-vapor interface. The vapor velocity is scaled by the factor $\epsilon$ as compared to the liquid velocity. Note that the vectors are normalized differently in each panel.

Each panel takes a different normalization of the velocity vectors for the sake of a better view. The velocity pattern in the vapor phase is similar to that shown in Fig. 2 except that the direction of flow is opposite, namely, evaporation and condensation occur at the crest and the trough of the liquid phase, respectively. The most remarkable fact seen in Fig. 7 is the presence of vortices in the liquid phase. Two vortices rolling in opposite directions emerge within one hump of the liquid phase which resembles the velocity field inside a liquid film subjected to the Marangoni effect. ${ }^{30,31,37}$ However, in the present system, the vortices in the liquid phase shown in Fig. 7 are not induced by thermocapillarity, because setting the Marangoni number to zero yields almost the same velocity field (not shown).

To reveal the cause of this flow, we focus on the explicit expression for the horizontal component of the liquid veloc- 
ity at the liquid-vapor interface without the Marangoni effect $(M=0)$, derived from Eqs. (32) and (33) of Ref. 24,

$$
\left.u_{l}\right|_{z=h}=-\frac{1}{2} h(1+d) \partial_{x} p_{v}-\frac{1}{2} G h^{2} \partial_{x} h+\frac{1}{2} S h^{2} \partial_{x}^{3} h .
$$

For a steady state $\left(\partial_{t} h=0\right)$, the total liquid flow across the film vanishes if there is no mean flow from Eq. (1a),

$$
q_{l}=-\frac{h+3(1+d)}{12} h^{2} \partial_{x} p_{v}-\frac{h^{3}}{3} \partial_{x}\left(G h-S \partial_{x}^{2} h\right)=0 .
$$

Eliminating the gravity and surface-tension terms from Eq. (14) and substituting them into Eq. (13) yields

$$
\left.u_{l}\right|_{z=h}=-\frac{1}{8} h(1+d-h) \partial_{x} p_{v}=\frac{3 \eta h q_{v}}{2(1+d-h)^{2}},
$$

where we have used Eq. (2c). The sign of $\left.u_{l}\right|_{z=h}$ is the same as that of $q_{v}$, which indicates that the liquid flows in the same direction as the vapor near the interface. This is consistent with the velocity fields shown in Fig. 7. Thus, the flow in the liquid phase near the interface originates from the vapor flow, which exerts a shear stress along the interface. As in the case of the Marangoni convection, this shear stress gives rise to the bulk liquid flow near the interface through the viscosity of the liquid toward the trough of the liquid phase where condensation takes place. To compensate for the mass balance, there must be also a counterflow of the liquid near the wall. Thus, the vortices in the liquid phase arise from the viscous coupling with the vapor flow. This situation is in sharp contrast with that considered in the study of bilayer convection, ${ }^{38,39}$ where only a liquid viscously drags the other fluid because gases are too tenuous to exert much shear. Furthermore, the emergence of convective cells in the liquid layer of bilayer systems with phase change and without Rayleigh convection ${ }^{18,22,33}$ suggests that this vapor-flowdriven mechanism arising from evaporation or condensation occurs instead of that of thermocapillarity, which is now suppressed by the phase-change effect. It is worthwhile noting that in our theory the tangential velocity of the vapor at the interface vanishes at the leading-order approximation, see Eq. (27c) in Ref. 24, because the tangential liquid and vapor velocities must balance at the interface as it follows from the no-slip boundary condition [Eq. (21c) of Ref. 24], whereas the vapor velocity has been assumed to be one order lower in $\epsilon$ than the liquid velocity as in the scaling (23) in Ref. 24. To circumvent this inconsistency, we have set the tangential component of the vapor velocity at the interface to zero at leading order, while at next order the vapor velocity is equal to that of the leading-order liquid velocity. Hence, the leading-order liquid flow shown in Fig. 7 is formally driven solely by the shear stress, not by the viscous drag, exerted by the leading-order vapor flow.

The time variation of the maximal and minimal values of the vapor pressure for different evaporation numbers is presented in Fig. 8(a). Due to the fact that the vapor pressure is almost uniform in space compared to the variation of its absolute value, these two values are too close to each other to be discernible. We also find that the pressure variation is not monotonic with respect to the evaporation number $E$. The dependence of the maximal vapor pressure of the steady state
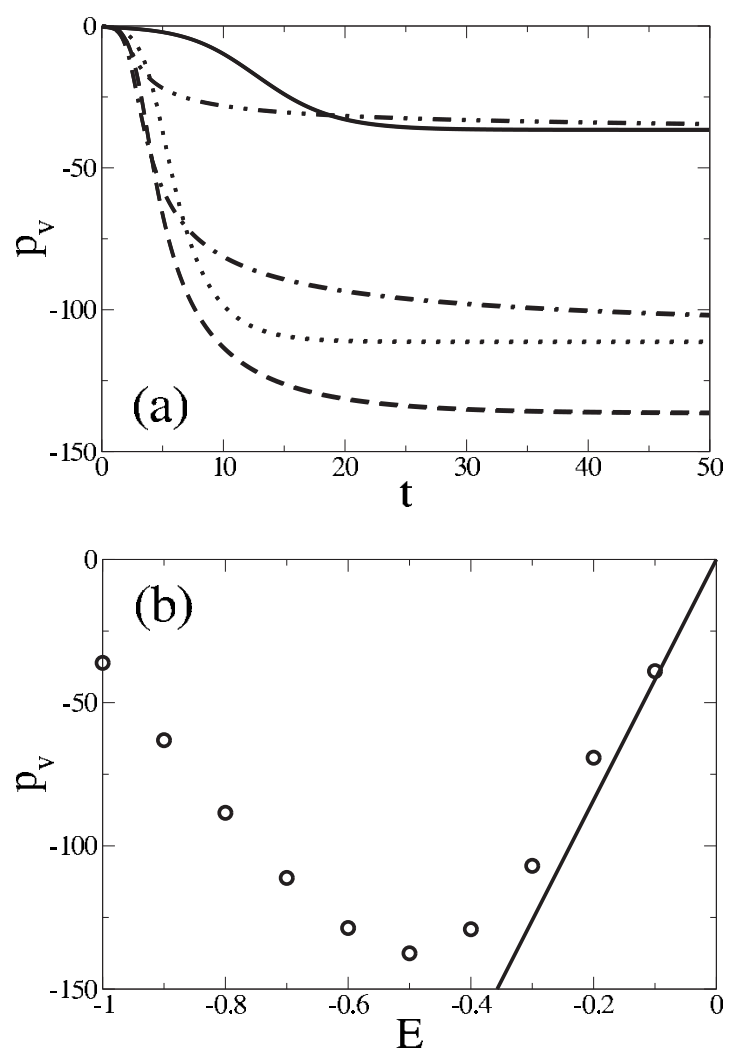

FIG. 8. Vapor pressure $p_{v}$ in the case of Rayleigh-Taylor instability for $d=2$. (a) Temporal evolution of $p_{v}$. Although spatially nonuniform, its variation is small on the graph scale. The solid, dotted, dashed, dotted-dashed, and two-dotted-dashed curves correspond to $E=-1,-0.7,-0.5,-0.3$, and -0.1 , respectively. (b) Variation of the maximal vapor pressure of the steady state with the evaporation number $E$. The solid line represents the relationship $p_{v}=\rho E / \Pi$.

on the evaporation number is displayed in Fig. 8(b). The maximal pressure variation is achieved when $E=-0.5$. In the cases presented here, the vapor pressure variations are larger than that of the superheated or supercooled case, as displayed in Fig. 3.

The variation of the minimal and maximal values of the interfacial temperature follows the same tendency as that of the vapor pressure shown in Fig. 8, because the approximate Eq. (12) also holds in this case. Figure 9 presents the profiles of the interfacial temperature $T_{I}$ in the steady state along with those of the saturation temperature variation $\Delta T_{s}$. Note that since we have scaled the temperature with $\Delta T$, which is negative for the system heated from the vapor side [see Eq. (7)], positive values of $T$ represent the temperature lower than its equilibrium value and higher values of $T$ indicate cooler parts of the system. Both interfacial temperature and saturation temperature variation exhibit a trimodal variation consistent with the structure of the interface with crests and troughs of the interphase boundary, shown in Fig. 6(a) corresponding, respectively, to the maxima and minima of the temperatures presented in Fig. 9. In the case of $E=-1$ shown in Fig. 9(a), both of the temperature profiles are almost sinusoidal. In the case of $E=-0.5$, the crests of the interface temperature become elongated, while those of the saturation temperature variation remain rounded. In the case of $E=-0.1$, the elongated crests of the interface temperature 

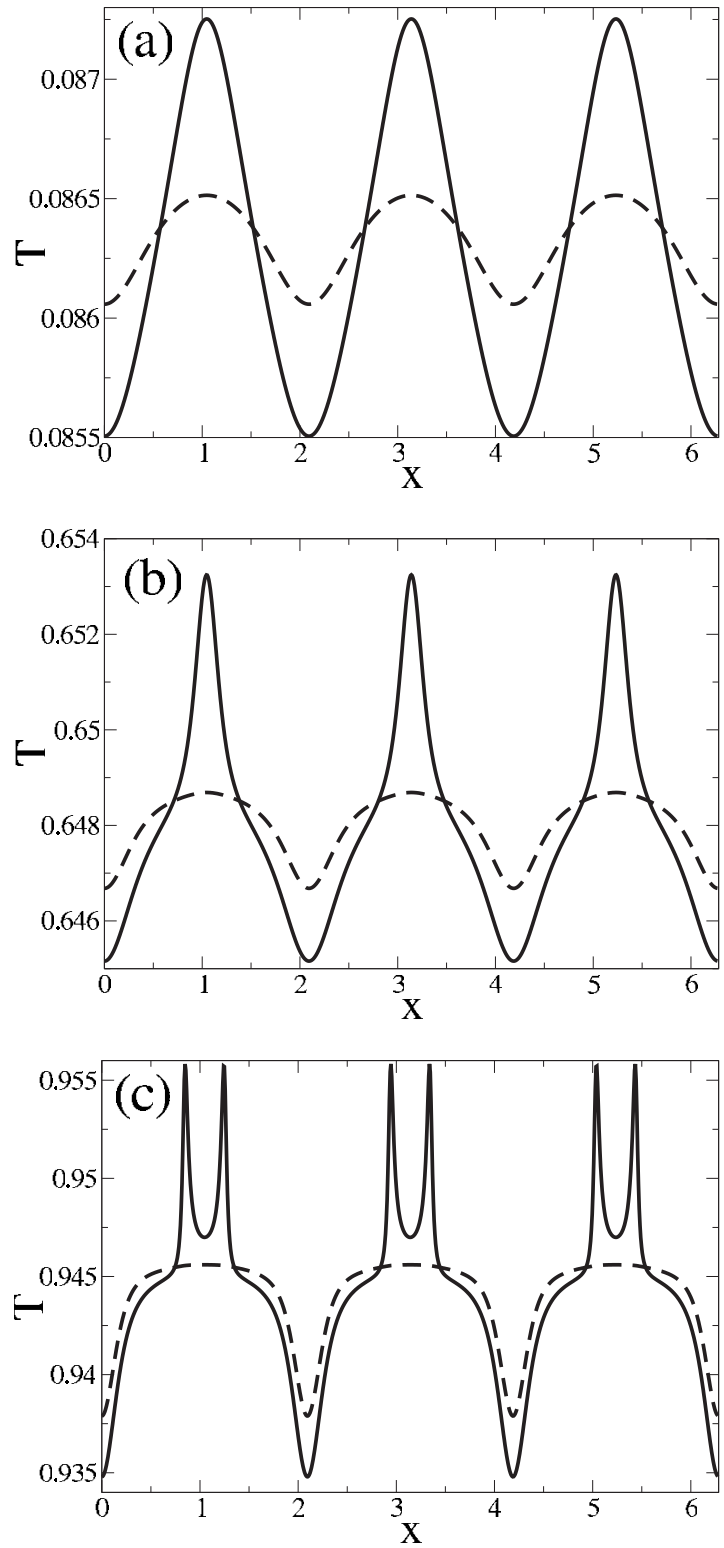

FIG. 9. Spatial variation of the interface temperature (solid curve) and the saturation temperature variation (dashed curve) in the steady state for $d=2$. (a) $E=-1$, (b) $E=-0.5$, and (c) $E=-0.1$.

split into two fingers which corresponds to the emergence of the secondary drops in the depressions of the interphase boundary. It must be noted that in spite of visually sharp structures that appear in the case of $E=-0.1$, the temperature gradients are still small given a difference in scales of the axes. This suggests the lesser importance of the Marangoni effect which will be discussed in Sec. VI. The difference between the shapes of the two curves implies that the interface temperature is quite sensitive to the presence of very thin regions in the liquid layer, whereas such sensitivity of the saturation temperature variation or equivalently the vapor pressure is weak. For all the cases in Fig. 9, $T>0$, and therefore the temperature is below its equilibrium value. However, since the temperature must not be lower than that of the liquid-side cooled plate, $T<1$ must hold. If we apply this condition to the saturation temperature variation, the constraint for the vapor pressure, $p_{v}>\rho E / \Pi(E<0)$, is ob-
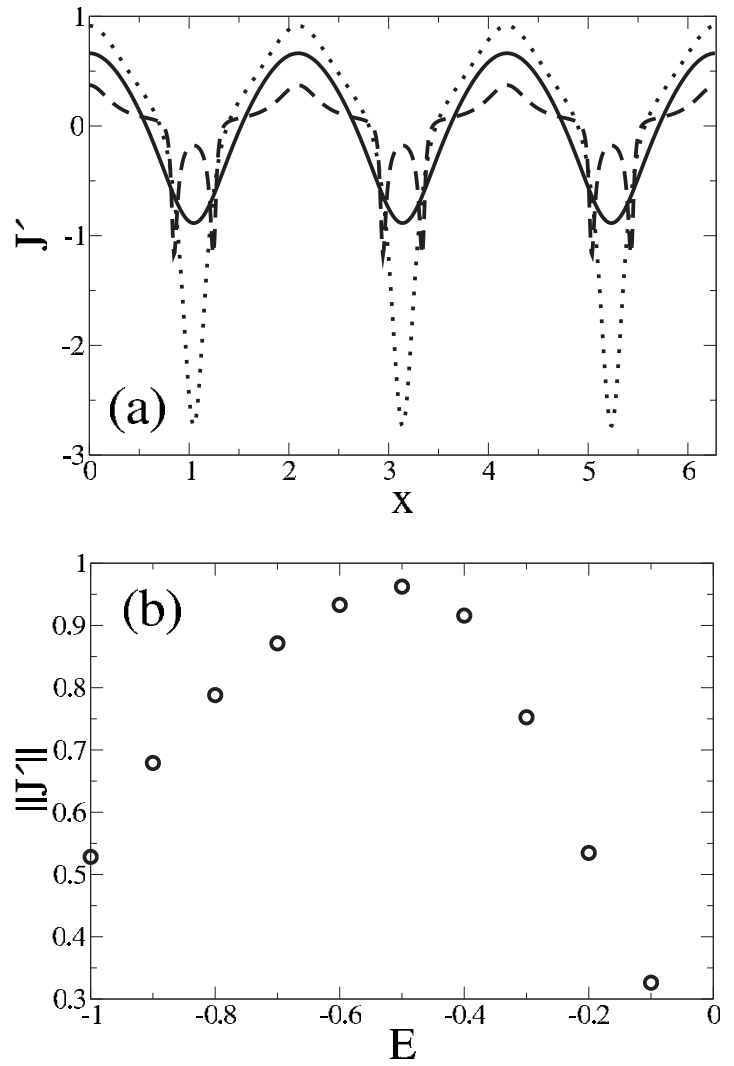

FIG. 10. Variation of the mass flux across the interface at the steady state. Positive and negative values of $J^{\prime}$ represent evaporation and condensation, respectively. (a) Spatial variation of $J^{\prime}$ for $E=-1$ (solid curve), -0.5 (dotted curve), and -0.1 (dashed curve). (b) Variation of the root mean square of $J^{\prime}$, Eq. (16), with the evaporation number $E$.

tained. In Fig. 8(b), we show the curve $p_{v}=\rho E / \Pi$. We find that the vapor pressure approaches this line as $|E|$ decreases. Thus, the vapor pressure increases as the interface approaches the cold plate, and attains its minimal value at $E$ $=-0.5$.

Spatial variation of the dimensionless mass flux across the interface is shown in Fig. 10(a), where the value of $J^{\prime}$ $=E J$ is displayed. Since $J$ is nondimensionalized by $\lambda_{l} \Delta T / d_{l} L, J^{\prime}$ is independent of $\Delta T$. The use of $J^{\prime}$ instead of $J$ is equivalent to scaling the mass flux by $\eta_{l} / d_{l}$. Here, positive (negative) values of $J^{\prime}$ represent evaporation (condensation). The profiles of the mass flux can be inferred from Fig. 9 , because it is derived from the difference between the two respective curves there, as discussed in the superheated or supercooled case. To measure the intensity of phase change, we introduce the root mean square of the mass flux in the steady state as

$$
\left\|J^{\prime}\right\|=\sqrt{\frac{1}{2 \pi} \int_{0}^{2 \pi} J^{\prime 2} d x,}
$$

and evaluate it versus the evaporation number $E$ in Fig. 10(b). A maximal value of $\left\|J^{\prime}\right\|$ emerges at $E=-0.5$. Therefore, phase change is most intensive at this value of $E$. For smaller values of $|E|$, the value of $\left\|J^{\prime}\right\|$ 
decreases because the temperature difference across the layers itself decreases. On the other hand, the interface is not sufficiently deformed to evaporate or condense at a significant rate for larger values of $|E|$.

\section{DISCUSSION: WHY DOES THE AVERAGE VAPOR PRESSURE VARY?}

As we observed in Figs. 3 and 8, the average vapor pressure over the period varies and deviates from zero during the evolution of the system. Moreover, it behaves differently in the superheated/supercooled and Rayleigh-Taylor unstable cases, and also for various values of the evaporation number in the latter case. Since the interface temperature evolves similarly to the vapor pressure, as we showed above, the deviation of the average interface temperature from its equilibrium value accompanies that of the average vapor pressure. In this section, we show the origin of this behavior.

First, as mentioned above, the total mass of the liquid phase over the period is conserved under periodic boundary conditions, as it follows from the gradient form of Eq. (1b). Therefore, the total mass flux over the period vanishes. Consequently, the integral of the right-hand side of Eq. (2a) must also vanish,

$$
\begin{aligned}
\int_{0}^{l} J^{\prime} d x= & -\int_{0}^{l} \frac{K}{1+K h+\frac{\lambda h}{1+d-h}} \frac{E(1+d)(h-1)}{1+d-h} d x \\
& -\int_{0}^{l} \frac{K}{1+K h+\frac{\lambda h}{1+d-h}} \\
& \times\left(1+\frac{\lambda h}{1+d-h}\right) \frac{\Pi}{\rho} p_{v} d x=0 .
\end{aligned}
$$

For simplicity, taking the limit of $K \rightarrow \infty$ and $\lambda \rightarrow 0$ (for the reference, in our system $\lambda=0.037$ ), Eq. (17) reduces to

$$
\int_{0}^{l} \frac{E(1+d)(h-1)}{h(1+d-h)} d x+\int_{0}^{l} \frac{\Pi p_{v}}{\rho h} d x=0 .
$$

Equation (18) provides an implicit relationship between the film thickness and the vapor pressure. For the flat interface $h=1$, the average vapor pressure vanishes, as it follows from Eq. (18). However, the average vapor pressure obtains a nonzero value when the interface becomes corrugated. Note that the first term of Eq. (18) contains the evaporation number. Therefore, the average vapor pressure varies with the evaporation number and the sign of the average vapor pressure is different in the superheated/supercooled and RayleighTaylor unstable cases. In the former, the first term of Eq. (18) becomes very large near the vapor-side wall $(h \sim 1+d)$. In this case, the first term of Eq. (18) tends to be positive in the vicinity of the vapor layer rupture, leading to the sudden drop of the vapor pressure shown in Fig. 3. Nevertheless, the point $h=1+d$ as well as $h=0$ is not a singular point because of the finite and nonzero respective values of $K$ and $\lambda$, as can be seen from Eq. (17). Note that the variation of the average vapor pressure is inversely proportional to the parameter $\Pi$ representing the effect of the local saturation temperature variation. Thus, the evolution of the average vapor pressure arises from mass conservation in the vapor phase and the variation of the local saturation temperature, and depends on the evaporation number and the functional form of $h$ which depicts the shape of the interface.

\section{EFFECTS OF THERMOCAPILLARITY AND DEGREE OF NONEQUILIBRIUM}

In the numerical investigations discussed above, we have included the thermocapillary effect in the set of Eqs. (8) and (3). However, the recent full linear stability analyses ${ }^{18,21-23}$ of liquid-vapor bilayer systems suggest that this effect is negligible in the presence of phase change at the interface, because the large latent heat of vaporization makes the interface almost isothermal, and therefore the resulting temperature gradient along the interface becomes too small to induce a significant thermocapillarity. Moreover, in the linear stability theory of the present model, ${ }^{24}$ the Marangoni effect is found to be quantitatively very small, as compared to other effects for the system considered here if $K \gg 1$. In this section, we evaluate the contribution of the Marangoni effect in the nonlinear regime using the numerical solution of Eqs. (8) and (3). We also investigate the dependence of thermocapillarity on the accommodation coefficient, since the rate of evaporation or condensation decreases with smaller values of either $K$ or $\alpha$. Hence, the temperature homogenization effect of latent heat on the interface mentioned above becomes weaker, thereby leading to the enhancement of the Marangoni effect. In the aforementioned studies, ${ }^{18,21-23}$ the thermodynamic equilibrium was assumed at the liquid-vapor interface and the effect of the degree of nonequilibrium was not investigated. However, recently this effect was included and studied by Liu and Liu. ${ }^{33}$

In Fig. 11, we assessed the effects of thermocapillarity and of the degree of nonequilibrium. Figure 11(a) displays the variation of the rupture time of the vapor layer with the accommodation coefficient in the superheated/supercooled case. For $\alpha=1$ and 0.1 , there is no significant difference whether or not the Marangoni effect is taken into account. A slight difference appears in the rupture times for $\alpha=0.01$. For $\alpha=0.001$, this difference becomes large. The rupture time of the vapor layer without thermocapillarity is larger than that with thermocapillarity, which is obvious from the fact that the Marangoni effect is destabilizing when the liquid side is heated. In Fig. 11(b), we show the variation of the maximal and minimal film thicknesses of the stationary state for $E=-1$ in the case of the Rayleigh-Taylor instability. As in Fig. 11(a), the significant difference appears for $\alpha=0.01$, which increases at $\alpha=0.001$. The amplitude of the interface deformation becomes large as $\alpha$ decreases in the case without thermocapillarity. On the other hand, in the presence of thermocapillarity, the amplitude remains almost the same and even slightly shrinks due to the stabilizing impact of the Marangoni effect. ${ }^{30,36,40}$ For both the superheated/supercooled and Rayleigh-Taylor unstable cases, the Marangoni effect is negligible for larger values of the accommodation coefficient, e.g., $\alpha>0.1$, which correspond to the cases near the thermodynamic equilibrium, 

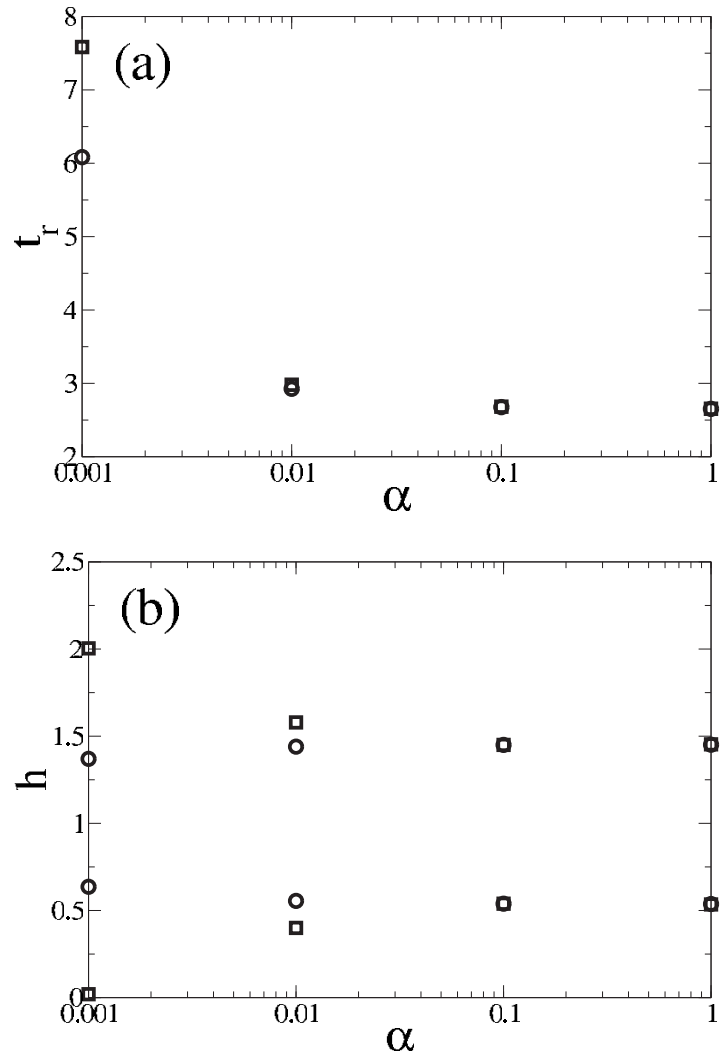

FIG. 11. The effects of thermocapillarity and of the degree of nonequilibrium. (a) Variation of the rupture time of the vapor layer with the accommodation coefficient $\alpha$. (b) Variation of the maximal and minimal film thicknesses of the stationary state in the Rayleigh-Taylor unstable case for $E=-1$ with the accommodation coefficient $\alpha$. In both panels, circles and squares represent the corresponding values with and without the Marangoni effect, respectively.

whereas the Marangoni effect becomes significant for smaller values of the accommodation coefficient, e.g., $\alpha<0.01$, which correspond to the cases far from the thermodynamic equilibrium.

Figure 12 shows the dependence of the maximal and minimal values of the interfacial temperature $T_{I}$ and of the saturation temperature variation $\Delta T_{s}$ of the stationary state given by Eq. (12) on the accommodation coefficient $\alpha$ when the Marangoni effect is taken into account for the RayleighTaylor unstable case with $E=-1$. Although the interfacial shape in this case does not change significantly with the decrease of the accommodation coefficient as can be seen in Fig. 11(b), the distribution of the interface temperature changes drastically. For $\alpha>0.1$, the interface temperature is almost the same as the saturation temperature variation, which is not sufficient to cause thermocapillarity. For $\alpha<0.01$, the interface temperature departs from the saturation temperature variation and the difference between the maximal and minimal interfacial temperatures increases. Since the saturation temperature variation $\Delta T_{s}$ is proportional to the vapor pressure for $K \gg 1$ [see Eq. (12)], the discrepancy between the saturation temperature variation and the interface temperature indicates the break of similarity in the behaviors of the vapor pressure and the interface temperature. This result is expected based on the expression of

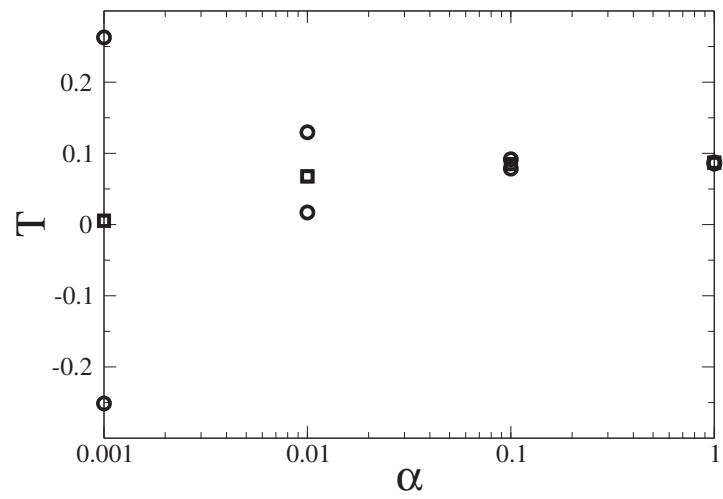

FIG. 12. Variation of the maximal and minimal values of the interfacial temperature $T_{I}$ (circles) and the saturation temperature variation $\Delta T_{s}$ in Eq. (12) (squares) of the stationary state for the Rayleigh-Taylor unstable case with $E=-1$ with the Marangoni effect taken into account.

the interface temperature $T_{I}$ given by Eq. (3) which suggests a weaker influence of the vapor pressure for smaller $K$. As the contribution of the vapor pressure in Eq. (3) decreases with the decrease in $\alpha$, the effect of the interface deformation expressed by the first term in the square bracket of Eq. (3) becomes dominant. Hence, the interface temperature is strongly affected by the interfacial deformation for smaller $\alpha$, which results in the enhancement of the Marangoni effect.

\section{CONCLUSION}

We have numerically investigated the nonlinear dynamics of a bounded thin liquid-vapor bilayer system in the framework of the model recently developed in Ref. 24. Here, we have considered the two cases: the first is that of the liquid (vapor) heated (cooled) and gravity acting toward the liquid (the superheated or supercooled case), and the second is that of the liquid (vapor) cooled (heated) and gravity acting toward the vapor (the Rayleigh-Taylor unstable case). In both cases, the effects of vapor pressure and gravity counteract each other: the former is destabilizing and the latter stabilizing in the superheated or supercooled case, while their roles are reversed in the Rayleigh-Taylor unstable case. In the superheated or supercooled case, neither a rupture of the liquid layer nor a steady state has been found for realistic sets of parameter values. The absence of a liquid rupture suggests an essentially different instability mechanism from that of a Marangoni-driven bilayer system. ${ }^{28,30}$ In contrast, nontrivial nonruptured stationary states emerge after a sufficiently long time in the Rayleigh-Taylor unstable case. In the stationary states, we have found vapor-flow-driven convective cells in the liquid phase. Moreover, the average vapor pressure is found to deviate from its equilibrium value once the interface departs from the flat equilibrium state. We have shown that this phenomenon can be directly derived from Eq. (8b). Since we have shown that the interface temperature is nearly equal to the saturation temperature variation, which is proportional to the vapor pressure, near the thermodynamic equilibrium limit, the evolution of the interface temperature follows that of the vapor pressure and the same deviation is also found for the former. The Marangoni effect does not appear near the thermodynamic equilibrium because 
the vapor pressure does not induce sufficient temperature variation along the interface. However, if the system becomes far from the thermodynamic equilibrium, the interface temperature is strongly affected by the interfacial deformation, which results in the emergence of the Marangoni effect.

In this paper, we restricted our attention to the spatiotemporal dynamics of the model as an initial value problem. Hence, we did not provide here a more comprehensive investigation on the bifurcation nature or the structure of steady states of the system and their stability based on the direct analysis of the governing equations (1) without the time-dependent term $\left(\partial_{t} h=0\right)$. Furthermore, variation of the domain periodicity would lead to another type of solutions, allowing one to look into the problem of wavelength selection. This work should deserve a separate paper.

In order to confirm whether our leading-order model is consistent, it is necessary to check that the higher-order terms are indeed much smaller than those of the leading order. In our case, one of the major assumptions is that a term representing mass loss or gain during phase change is negligible in Eq. (1a). This assumption is valid when the condition $\epsilon|E J| \ll\left|\partial_{t} h\right|$ is satisfied (see Ref. 24). Based on the present numerical analysis, we have confirmed this condition for the superheated or supercooled case. For the RayleighTaylor unstable case, however, this condition breaks down as the steady states are approached, since $\left|\partial_{t} h\right|$ must vanish there. Therefore, the steady solutions shown in this paper should be directly modified by the effect of mass loss or gain. To assess this effect precisely, numerical analysis of either a model including next-order terms or the full NavierStokes and energy equations should be carried out. We anticipate that this modification is small because this effect is connected with the variables of next order. Nevertheless, it may affect the value of the critical temperature difference at which the rupture of the liquid layer occurs.

In addition, we have also found another higher-order term which may violate our assumption. In the mass balance equation [Eq. (21a) of Ref. 24], we have neglected the interface velocity compared to the vapor velocity at the interface, and thereby derived Eq. (1b). For the Rayleigh-Taylor unstable case of smaller values of $|E|$, the former becomes comparable to the latter in the very beginning of the evolution. However, for the same reason mentioned above, this would not change the interface dynamics significantly. Furthermore, it cannot affect the long-time evolution or the stationary states.

Finally, the following remark is in order. The presence of vortices in the velocity field of the liquid may violate the assumption of the long-wave approximation wherever the vertical component of the velocity exceeds the horizontal one; recall scaling of the horizontal and vertical components of the liquid velocity as $\mathcal{O}(1)$ and $\mathcal{O}(\epsilon)$, respectively. ${ }^{24}$ The vortices in the framework of the long-wave theory were reported in Ref. 31 and also in a study of falling liquid films on corrugated surfaces. ${ }^{35}$ In Ref. 31, the authors justified its application on the grounds that a comparison with the fullscale numerical computations has shown a good agreement. Also in our case, such a comparison is expected to confirm the validity of the long-wave theory as well as to show whether the above-mentioned higher-order effects are indeed negligible.

\section{ACKNOWLEDGMENTS}

K.K.'s visit to the Technion-Israel Institute of Technology during which this work was started was financially supported by the Bilateral International Exchange Program (BIEP) of the Global COE "The Next Generation of Physics, Spun from Universality and Emergence" from the Ministry of Education, Culture, Sports, Science and Technology (MEXT) of Japan. The research was also partially supported by the European Union via the FP7 Marie Curie scheme [Grant No. PITN-GA-2008-214919 (MULTIFLOW)]. The authors also thank the referees for their critical and pertinent suggestions which helped to improve the quality of the paper.

\section{APPENDIX: LINEAR STABILITY CONDITION WITH THERMOCAPILLARITY FOR SMALL ACCOMMODATION COEFFICIENT}

In the previous paper, ${ }^{24}$ it was shown that thermocapillarity does not affect the linear stability threshold if the liquid-vapor interface is near local thermodynamic equilibrium, $K \gg 1$. However, the results of Sec. VI suggest that the importance of the Marangoni effect is strongly related to the degree of nonequilibrium, i.e., to the value of the accommodation coefficient $\alpha$. In this appendix, we discuss the influence of the thermocapillary effect on the linear stability threshold when the system significantly deviates from thermodynamic equilibrium.

For the superheated or supercooled case, the stability criterion is [see Eq. (48) of Ref. 24]

$$
\frac{G^{\prime} k_{0}^{2}}{3 A}>1
$$

where

$$
G^{\prime}=G-\frac{3}{2} \frac{1+d}{d} \frac{M}{1+K+\frac{\lambda}{d}},
$$

and $A$ and $k_{0}^{2}$ are defined in Eq. (10). In this case $(E, G>0)$, the Marangoni effect lowers the left-hand side of this inequality for $M>0$ regardless of the value of $K$. Since the condition equation (A1) is not satisfied for a realistic system (see Ref. 24), the Marangoni effect and the degree of nonequilibrium do not change the linear stability properties of experimentally feasible systems.

In contrast, the critical condition for the Rayleigh-Taylor unstable case is much more complicated. It reads [see Eq. (55) of Ref. 24] 

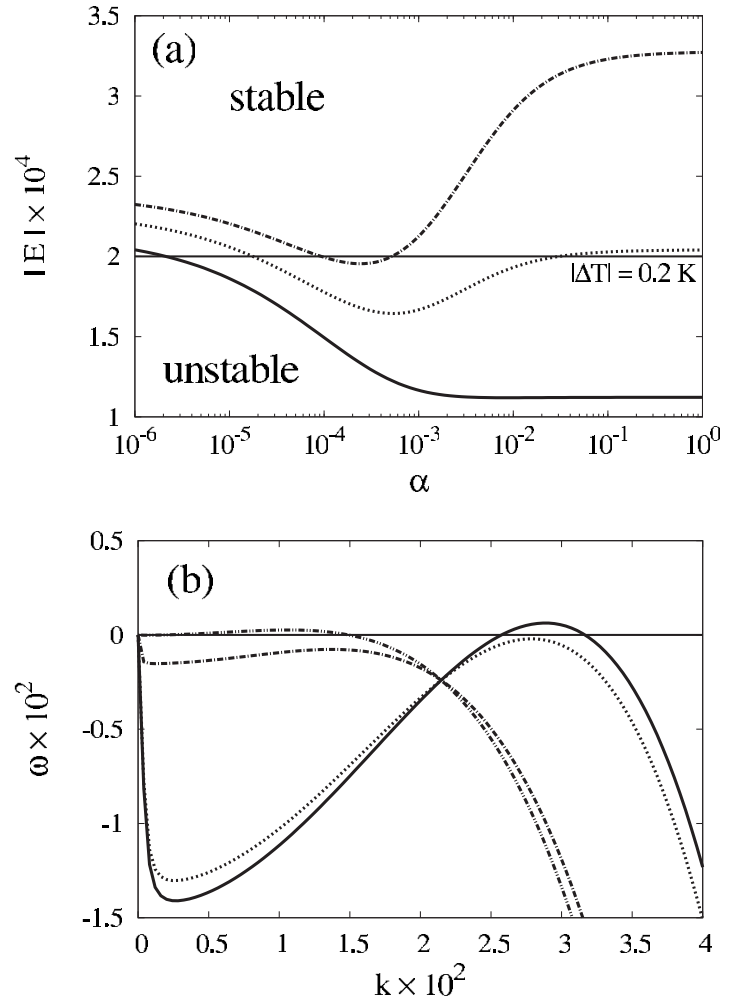

FIG. 13. Dependence on the accommodation coefficient $\alpha$. (a) Neutral stability curves for $d=2,2.5$, and 3 from bottom to top. The horizontal line corresponds to $|\Delta T|=0.2 \mathrm{~K}$. (b) Growth rate $\omega$ as a function of the disturbance wavenumber $k$ for $d=2.5$ and $|\Delta T|=0.2 \mathrm{~K}$. The curves correspond to $\alpha=1,10^{-2}, 10^{-4}$, and $10^{-6}$ from bottom to top in the range of small wavenumbers.

$$
\left\{\begin{array}{cc}
\frac{\left|G^{\prime}\right| k_{0}^{2}}{3|A|}<1 & \text { for } \frac{S k_{0}^{2}}{\left|G^{\prime}\right|}>1 \\
\frac{\left(\left|G^{\prime}\right|+S k_{0}^{2}\right)^{2}}{12|A| S}<1 & \text { for } \frac{S k_{0}^{2}}{\left|G^{\prime}\right|}<1
\end{array}\right.
$$

Since we seek the condition for the temperature difference $\Delta T$, the expressions of $|A|$ and $\left|G^{\prime}\right|$ can be recast as

$$
|A|=c_{1}|E|, \quad\left|G^{\prime}\right|=|G|-c_{2}|E|,
$$

with the coefficients $c_{1}, c_{2}$ independent of $\Delta T$,

$$
\begin{aligned}
& c_{1}=\frac{\eta(1+d)}{\rho d^{4}} \frac{K}{1+K+\frac{\lambda}{d}}\left(4+3 d+\frac{6|M| \Pi}{\rho|E|} \frac{K}{1+K+\frac{\lambda}{d}}\right) \\
& c_{2}=\frac{3}{2|E|} \frac{1+d}{d} \frac{|M|}{1+K+\frac{\lambda}{d}}
\end{aligned}
$$

Rewriting Eq. (A3) in terms of $|E|$ using Eq. (A4), the following stability condition is obtained:

$$
\left\{\begin{array}{l}
|E|>\frac{|G|}{3 c_{1} / k_{0}^{2}+c_{2}} \text { for }|E|>\frac{|G|-S k_{0}^{2}}{c_{2}} \\
|E|_{-}<|E|<|E|_{+} \text {for }|E|<\frac{|G|-S k_{0}^{2}}{c_{2}}
\end{array}\right.
$$

where

$$
|E|_{ \pm}=\frac{c_{2}\left(|G|+S k_{0}^{2}\right)+6 c_{1} S \pm 2 \sqrt{3 c_{1} S\left\{c_{2}\left(|G|+S k_{0}^{2}\right)+3 c_{1} S\right\}}}{c_{2}^{2}} .
$$

Since $\left(|G|-S k_{0}^{2}\right) / c_{2}<|E|_{+}$by definition, the condition $|E|<|E|_{+}$in the second line of Eq. (A6) is not necessary. Moreover, we numerically found that $|G| /\left(3 c_{1} / k_{0}^{2}+c_{2}\right)$ $\ll\left(|G|-S k_{0}^{2}\right) / c_{2}$ in the parameter range considered here. Therefore, the only stability condition left from Eq. (A6) is $|E|_{-}<|E|$.

The stability diagram in terms of $|E|$ and $\alpha$ plane is shown in Fig. 13(a). For the curve of $d=2$, the behavior is similar to those without the Marangoni effect shown in Fig. 5 of Ref. 24. However, compared to the counterpart $\left(d_{l}=1.0\right.$ $\left.\times 10^{-4} \mathrm{~m}\right)$, the flat region of the curve for large accommodation coefficients becomes wider due to the stabilizing effect of thermocapillarity. This effect even lowers the stability threshold in terms of $|E|$ for $d=2.5$ and 3 from their respective values at $\alpha=1$. In these cases, the threshold values of the evaporation number decrease as the accommodation coefficient becomes smaller for large accommodation coefficients, until they attain their minima at $\alpha=10^{-4} \sim 10^{-3}$. If the accommodation coefficient becomes further smaller, the threshold evaporation numbers increase as $d=2$. In the limit of $\alpha$ $\rightarrow 0$, the stability condition reduces to $|G|<c_{2}|E|$, which is identical with that of the Rayleigh-Taylor unstable bilayer system without phase change. ${ }^{29,30}$

In Fig. 13(b), we present the dispersion curves for $d$ $=2.5$ and $|\Delta T|=0.2 \mathrm{~K}$ for various values of $\alpha$. The shapes of the curves are quite different between $\alpha=1$ or $10^{-2}$, on one hand, and $\alpha=10^{-4}$ or $10^{-6}$, on the other hand. This suggests that there is an exchange of the instability mechanism between $\alpha=10^{-2}$ and $10^{-4}$. When the accommodation coefficient is large ( $\alpha>10^{-2}$ for this case), the vapor pressure effect due to evaporation or condensation is the main stabilizing mechanism against the destabilizing gravitational effect. At the minimum point of the threshold evaporation number shown in Fig 13(a) $\left(\alpha=10^{-3} \sim 10^{-4}\right)$, both the vapor pressure and Marangoni effects are significant. However, as $\alpha$ approaches $0\left(\alpha<10^{-4}\right)$, the former diminishes and the latter becomes dominant. As a result, the fastest growing wavenumber becomes consistent with that of the case with no phase change. ${ }^{29,30}$

\footnotetext{
${ }^{1}$ A. Oron, S. H. Davis, and S. G. Bankoff, "Long-scale evolution of thin liquid films," Rev. Mod. Phys. 69, 931 (1997), and references therein.

${ }^{2}$ R. V. Craster and O. K. Matar, "Dynamics and stability of thin liquid films," Rev. Mod. Phys. 81, 1131 (2009), and references therein.

${ }^{3}$ H. J. Palmer, "The hydrodynamic stability of rapidly evaporating liquids at reduced pressure," J. Fluid Mech. 75, 487 (1976).

${ }^{4}$ A. Prosperetti and M. S. Plesset, "The stability of an evaporating liquid surface," Phys. Fluids 27, 1590 (1984).

${ }^{5}$ F. J. Higuera, "The hydrodynamic stability of an evaporating liquid,"
} 
Phys. Fluids 30, 679 (1987)

${ }^{6}$ J. P. Burelbach, S. G. Bankoff, and S. H. Davis, "Nonlinear stability of evaporating/condensing liquid films," J. Fluid Mech. 195, 463 (1988).

${ }^{7}$ J. R. Maa, "Evaporation coefficients of liquids," Ind. Eng. Chem. Fundam. 6, 504 (1967); Also, J. R. Maa, "The role of interfaces in heat transfer processes," Adv. Colloid Interface Sci. 18, 227 (1983).

${ }^{8}$ A. Oron, "Three-dimensional nonlinear dynamics of thin liquid films," Phys. Rev. Lett. 85, 2108 (2000).

${ }^{9}$ A. Oron and S. G. Bankoff, "Dewetting of a heated surface by an evaporating liquid film under conjoining/disjoining pressures," J. Colloid Interface Sci. 218, 152 (1999).

${ }^{10}$ A. Oron and S. G. Bankoff, "Dynamics of a condensing liquid film under conjoining/disjoining pressures," Phys. Fluids 13, 1107 (2001).

${ }^{11}$ E. Sultan, A. Boudaoud, and M. B. Amar, "Evaporation of a thin film: Diffusion of the vapour and Marangoni instabilities," J. Fluid Mech. 543, 183 (2005)

${ }^{12}$ M. Bestehorn and D. Merkt, "Regular surface patterns on Rayleigh-Taylor unstable evaporating films heated from below," Phys. Rev. Lett. 97, 127802 (2006).

${ }^{13} \mathrm{M}$. Bestehorn, "Convection in thick and in thin fluid layers with a free surface-The influence of evaporation," Eur. Phys. J. Spec. Top. 146, 391 (2007).

${ }^{14}$ A. Huang and D. D. Joseph, "Instability of the equilibrium of a liquid below its vapour between horizontal heated plates," J. Fluid Mech. 242, 235 (1992).

${ }^{15}$ J. Margerit, P. Colinet, G. Lebon, C. S. Iorio, and J. C. Legros, "Interfacial nonequilibrium and Bénard-Marangoni instability of a liquid-vapor system," Phys. Rev. E 68, 041601 (2003).

${ }^{16}$ P. N. Shankar and M. D. Deshpande, "On the temperature distribution in liquid vapor phase change between plane liquid surfaces," Phys. Fluids A 2, 1030 (1990).

${ }^{17} \mathrm{G}$. Fang and C. A. Ward, "Temperature measured close to the interface of an evaporating liquid," Phys. Rev. E 59, 417 (1999).

${ }^{18} \mathrm{O}$. Ozen and R. Narayanan, "The physics of evaporative and convective instabilities in bilayer systems: Linear theory," Phys. Fluids 16, 4644 (2004).

${ }^{19}$ O. Ozen and R. Narayanan, "The physics of evaporative instability in bilayer systems: Weak nonlinear theory," Phys. Fluids 16, 4653 (2004).

${ }^{20}$ O. Ozen and R. Narayanan, "A note on the Rayleigh-Taylor instability with phase change," Phys. Fluids 18, 042110 (2006).

${ }^{21}$ G. B. McFadden, S. R. Coriell, K. F. Gurski, and D. L. Cotrell, "Onset of convection in two liquid layers with phase change," Phys. Fluids 19, 104109 (2007).

${ }^{22}$ W. Guo and R. Narayanan, "Interfacial instability due to evaporation and convection: Linear and nonlinear analyses," J. Fluid Mech. 650, 363 (2010).

${ }^{23}$ G. B. McFadden and S. R. Coriell, "Onset of oscillatory convection in two liquid layers with phase change," Phys. Fluids 21, 034101 (2009).

${ }^{24} \mathrm{~K}$. Kanatani, "Interfacial instability induced by lateral vapor pressure fluctuation in bounded thin liquid-vapor layers," Phys. Fluids 22, 012101 (2010).

${ }^{25}$ B. S. Tilley, S. H. Davis, and S. G. Bankoff, "Nonlinear long-wave stability of superposed fluids in an inclined channel," J. Fluid Mech. 277, 55 (1994).

${ }^{26}$ S. W. Joo and K. C. Hsieh, "Interfacial instabilities in thin stratified viscous fluids under microgravity," Fluid Dyn. Res. 26, 203 (2000).

${ }^{27}$ D. Merkt, A. Pototsky, M. Bestehorn, and U. Thiele, "Long-wave theory of bounded two-layer films with a free liquid-liquid interface: Short- and long-time evolution," Phys. Fluids 17, 064104 (2005).

${ }^{28}$ S. J. VanHook, M. F. Schatz, J. B. Swift, W. D. McCormick, and H. L. Swinney, "Long-wavelength surface-tension-driven Bénard convection: Experiment and theory," J. Fluid Mech. 345, 45 (1997).

${ }^{29}$ J. M. Burgess, A. Juel, W. D. McCormick, J. B. Swift, and H. L. Swinney, "Suppression of dripping from a ceiling," Phys. Rev. Lett. 86, 1203 (2001).

${ }^{30}$ A. Alexeev and A. Oron, "Suppression of the Rayleigh-Taylor instability of thin liquid films by the Marangoni effect," Phys. Fluids 19, 082101 (2007).

${ }^{31}$ T. Gambaryan-Roisman and P. Stephan, "Thermocapillary convection and interface deformation in a liquid film within a micro-slot with structured walls," Microfluid. Nanofluid. 3, 207 (2007).

${ }^{32}$ C. H. Panzarella, S. H. Davis, and S. G. Bankoff, "Nonlinear dynamics in horizontal film boiling," J. Fluid Mech. 402, 163 (2000).

${ }^{33}$ R. Liu and Q. S. Liu, "The convective instabilities in a liquid-vapor system with a non-equilibrium evaporation interface," Microgravity Sci. Technol. 21, 233 (2009).

${ }^{34} \mathrm{~A}$. Oron, "Nonlinear dynamics of three-dimensional long-wave Marangoni instability in thin liquid films," Phys. Fluids 12, 1633 (2000).

${ }^{35} \mathrm{~A}$. Oron and C. Heining, "Weighted-residual integral boundary-layer model for the nonlinear dynamics of thin liquid films falling on an undulating vertical wall," Phys. Fluids 20, 082102 (2008).

${ }^{36}$ A. Oron and P. Rosenau, "Formation of patterns induced by thermocapillarity and gravity," J. Phys. II 2, 131 (1992).

${ }^{37}$ A. Oron and P. Rosenau, "On a nonlinear thermocapillary effect in thin liquid layers," J. Fluid Mech. 273, 361 (1994).

${ }^{38}$ D. Johnson and R. Narayanan, "Geometric effects on convective coupling and interfacial structures in bilayer convection," Phys. Rev. E 56, 5462 (1997).

${ }^{39}$ D. Johnson and R. Narayanan, "Marangoni convection in multiple bounded fluid layers and its application to materials processing," Philos. Trans. R. Soc. London, Ser. A 356, 885 (1998).

${ }^{40}$ R. J. Deissler and A. Oron, "Stable localized patterns in thin liquid films," Phys. Rev. Lett. 68, 2948 (1992). 